\title{
Marine Aquarium Temperature Controller and the IoT (Internet of Things)
}

\begin{abstract}
This design monitors and controls the temperature in a marine aquarium. This system measures the water temperature in an aquarium and then uses a heater and cooling fan to control the temperature within a range of (25-27) ${ }^{\circ} \mathrm{C}$. The measured information is displayed on ThingSpeak which is an Internet of Things (IoT) service. In this way the user can access this information from any smart device. ThingSpeak is an IoT analytics platform that allows the user to analyse and visualize data that was collected. In the design additional features such as water level sensing and LED lights control are also included. The device measures the aquarium water temperature and in addition to showing it on "ThingSpeak" displays it on an LCD screen. Ambient room temperature and humidity are measured and displayed on an OLED screen in addition to ThingSpeak. This design is a compact design with most of the boards being interconnected modules.
\end{abstract}

Keywords-Aquarium, Arduino Uno, Internet of Things (IoT), ThingSpeak, Temperature Controller.

\section{INTRODUCTION}

Marine aquariums are a beautiful way to bring nature into a household or business. These marine aquariums contain a wide variety of exotic fish species, plant life and corals. The price of aquariums varies widely. Marine hobbyists spend a lot of time, money and effort in successfully monitoring and controlling water temperature [1] within a range of (25-27) ${ }^{\circ} \mathrm{C}$. This parameter is important for running a healthy aquarium. The main issue that can occur during the operation of aquariums is malfunctioning of the water heater. The water temperature could rise well above $28^{\circ} \mathrm{C}$ if the heater remains on permanently. The heater could fail due to poor manufacturing, incorrect installation, user error or electrical fault. All heat sources are thermostatically controlled, but due to age and working conditions, the thermostat can break down causing the heater to remain on. There are many aquarium temperature monitoring and controlling devices in the market with the ability to access the aquarium through the internet. The problem with such devices is that they are expensive and not every household can afford such a device. If the temperature in the marine aquarium fluctuates a good temperature controller can be used to correct the temperature.
Marine aquarium temperature can be monitored remotely with internet connectivity [2], this will allow the user to remotely access the temperature information and make decisions if needed. Thus acting as soon as possible can save animal and plant life in the aquarium. Common heater control problems in marine aquariums and the expensive cost of these devices led to the design of this specific marine aquarium temperature controller with internet connectivity. This design will monitor the aquarium water temperature and ambient room temperature and display the information on an LCD and OLED screen [3]. If a problem occurs such as temperature fluctuations, the system has controls for the user to enable heating and cooling functions. Today's world revolves around the internet. Therefore, this system displays the information locally on an LCD, however the temperature information in the marine aquarium can be accessed from any device, anywhere using the Internet of Things (IoT) [2]. This can be achieved by using the ESP8266 Wi-Fi [4] module plugged to an Arduino Uno microcontroller [5]. Additional features in this design include LED lighting ON/OFF control for the aquarium. This design is cost effective and gives advanced features when compared to devices in the same price class category. Any other monitoring, measuring or displaying features can be added on and viewed on the internet for data capturing of conditions in the marine aquarium. The design can be adapted according to the customers needs and requirements, making it a universal application in the aquarium industry. The design is cost-effective and can accurately convey information using the latest technology in the Internet of Things (IoT) [2] application for aquariums. The paper is divided into section I, Introduction, section II, methodology, section III, results and discussion and the conclusion is given in section IV.

\section{Methodology}

The block diagram in Figure 1 shows the complete system design of the marine aquarium temperature controller. The design is sub divided into six sections namely; Wi-Fi module, LCD and OLED [3] screen, sensors, relay module, Arduino Uno and the power supply. 


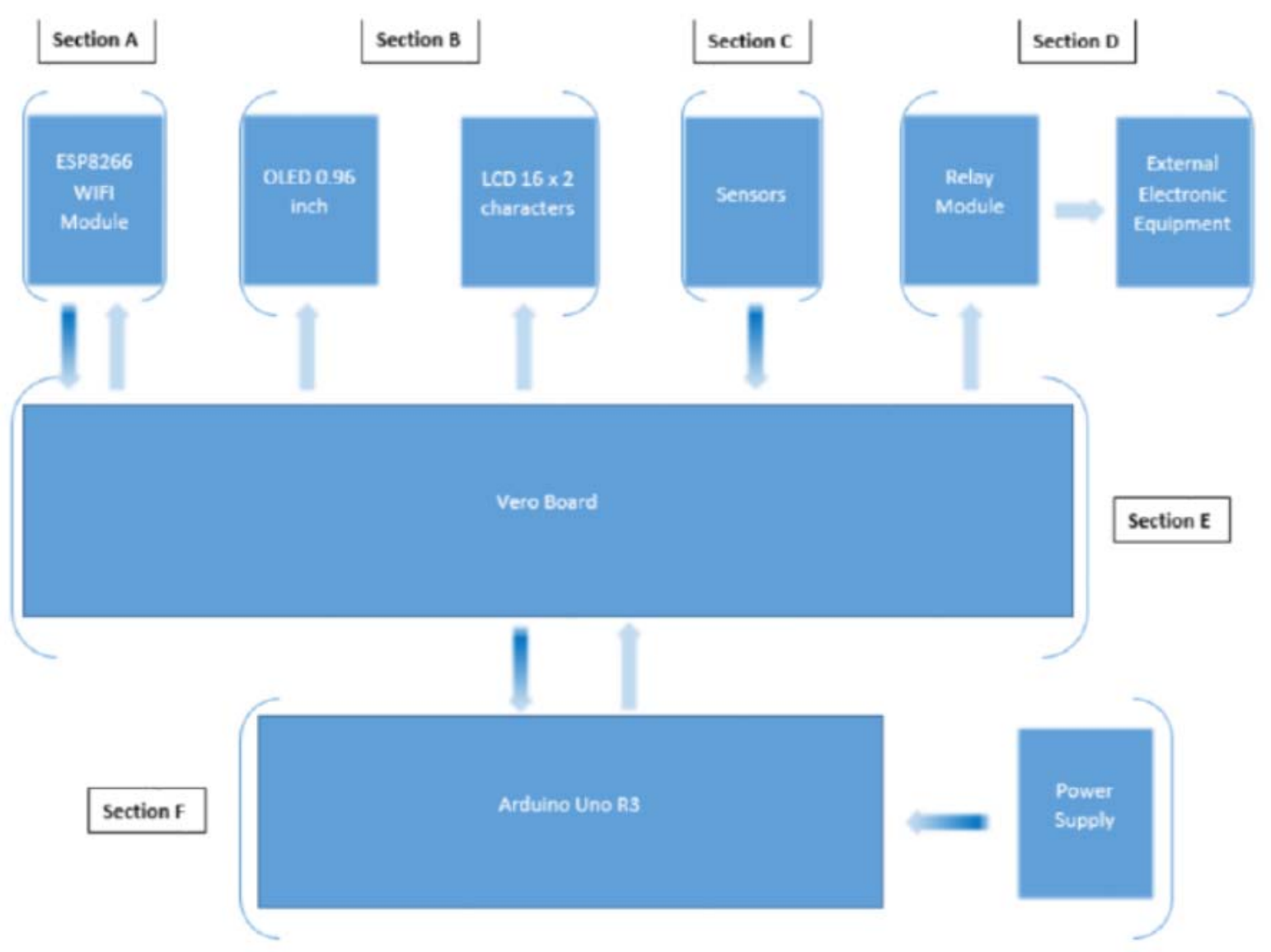

Fig. 1 Block diagram of the Marine aquarium temperature controller design

\section{A. Wi-Fi Module and IoT}

The ESP8266 Wi-Fi module communicates with the Arduino Uno using the UART which transmits and receives serial data from the Arduino Uno microcontroller [5]. In order to communicate with the ESP8266 Wi-Fi [4] module, the microcontroller uses a set of AT (Attention) commands. When the microcontroller communicates with the ESP8266 Wi-Fi module the Baud rate is specified as 115200 baud. A full list of AT commands can be accessed from the internet. The Wi-Fi module communicates with a router that provides access to the internet. The IoT platform used to view the data read by the sensors is ThingSpeak. The data is transmitted through the Wi-Fi module to the IoT server provider. The user creates an account with ThingSpeak, after which the data collected is displayed and then analyzed. The Wi-Fi module is set up as a TCP client to send and receive data. The Arduino Uno collects all the data measured from the different sensors, conveys this information through AT commands to the Wi-Fi module. The Wi-Fi module then sends the information through the router to the ThingSpeak server.

\section{B. LCD and the OLED display}

The LCD displays the measured aquarium temperature so that the user can physically see what the temperature is. Additional information can be added unto the display if the client requires any other information. The LCD interface uses eight lines to display the information, four lines for data, one line for enable, one line for read or write, one for $V_{C C}$ and one for $V_{D D}$. The contrast is adjusted by an externally mounted potentiometer. Two lines control the backlight of the LCD, these are connected to the Anode and Cathode of the LCD. The OLED screen displays the ambient temperature. It uses four connections which are $V_{C C}, V_{D D}$, SDA and SCL. This screen uses $I^{2} C$ [6] as a communication protocol. The $I^{2} C$ combines the best features of SPI and UARTs. The $I^{2} C$ enables the connection of multiple slaves to a single master (like SPI). In addition, you can have multiple masters controlling a single slave or multiple slaves. The $I^{2} C$ protocol data is transferred in messages. Messages are broken up into frames of data. Each message has an address frame that contains the binary address of the slave, and one or more data frames that contain the data being transmitted. The message also includes start and stop conditions, read/write bits and ACK/NACK bits between each data frame. Therefore, making the screen suitable for this design and application.

\section{Sensors and Veroboard}

The Vero board interfaces the connectors on the Arduino Uno board to the sensors as well as enables control to the relay module. Additional electronic components are mounted on the Vero board. This board acts as an interface between Arduino Uno and the external components. The 
sensors [7] measure the temperature and give an analogue voltage feedback to the Arduino. These values are converted into the correct format for display on the LCD. The Arduino Uno has 10-bit ADC channels used for this conversion.

\section{Relay Module and Peripheral equipment}

The relay [8-9] module controls the power to the electronic equipment. The Arduino will engage or disengage a specific relay according to the sensor information or the user decision on the IoT platform called ThingSpeak. The relay module consists of six relays on one module. Three of these relays where used, one for the heat source, one for the cooling fan and one to control the LED lights. These equipment need 220VAC, therefore a relay module capable of handling 220VAC and $10 \mathrm{Amp}$ maximum current was used.

\section{E. Arduino Uno and Power Supply}

The power supply is a compact unit bought off the shelf. The power supply converts $220 \mathrm{VAC}$ to $7.5 \mathrm{VDC}$ at $1 \mathrm{~A}$. The Arduino board has on board regulators to regulate voltages needed by the Arduino. The Ardunio Uno has two inputs for power supply. One is built for $5 \mathrm{~V}$ and the second $3.3 \mathrm{~V}$. The $5 \mathrm{~V}$ supply uses a LM7805 regulator and the 3.3 V uses a LT1117 - 3.3 regulator. The output from the power supply can vary between $(7.5-12)$ VDC.

\section{EXPERIMENTS AND RESULTS}

The block circuit diagram is divided into two connections. The first set of connections show the wiring layout from the Arduino Uno to the LCD and OLED displays. The second set of connections show the wiring layout of the Arduino Uno to the sensors, ESP8266 Wi-Fi module and the relays.

\section{A. Arduino Uno to LCD and OLED Displays}

The Arduino Uno is connected to the LCD and OLED displays via a breadboard as can be seen in the circuit diagram of Figure 2.

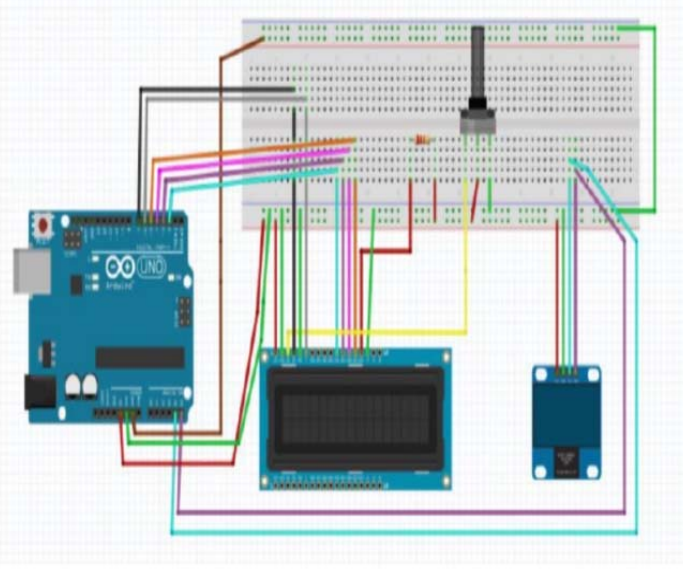

Fig. 2 Arduino, LCD and OLED connection
The LCD data lines are connected to the digital pins of the Arduino. The $V_{S S}$ and $V_{D D}$ on the LCD are connected to the Arduino power lines. The contrast pin of the LCD is connected to the potentiometer. This enables the user to adjust the contrast of the LCD according to the user's preference. The LCD backlight is connected to $V_{S S}$ through a $220 \Omega$ resistor for current limiting. The sensors measure different parameters such as temperature and the Arduino samples the values. The program used to code the Arduino converts the sampled values into actual parameter values which are then sent out as data to the four digital lines of the LCD. This controls the character the Arduino writes to the LCD. The OLED display only uses four lines for connection to the Arduino. The OLED uses $I^{2} C$ protocol for communication as described in section $\mathrm{B}$. The SDA and SCL lines are connected to the Arduino. The measured values are sent to the OLED display via the $I^{2} C$ protocol.

The wiring layout of the circuit diagram that could be used for PCB connections can be seen in Figure 3. The LCD display shows the water temperature and water level in the aquarium while the OLED is used to show the ambient room temperature and humidity in the final device.

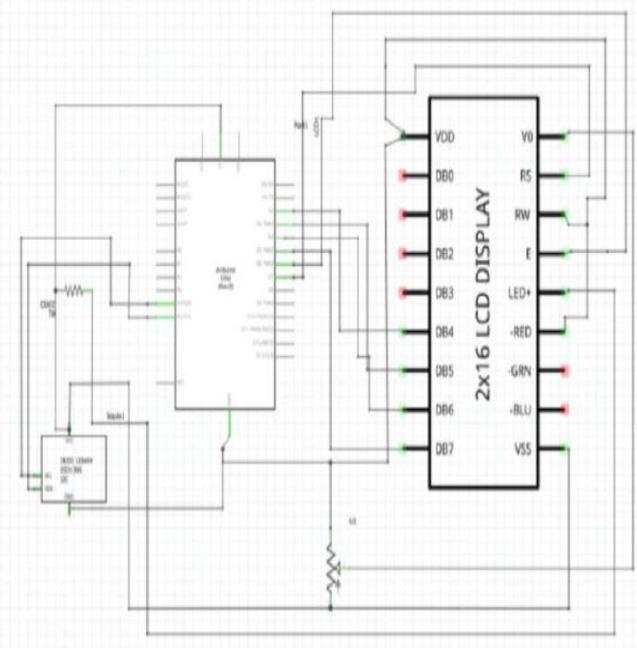

Fig. 3 Arduino with LCD and OLED connection wiring layout

\section{B. Arduino Uno to Sensors and Wi-Fi Module}

This section shows the design that connects the Arduino Uno to the sensors, ESP8266 Wi-Fi module and the relays. The ESP8266 Wi-Fi module uses UART communication basically serial communication. The TX and RX lines on the Wi-Fi module are connected to the RX and TX pins on the Arduino. The $V_{D D}$ and $V_{S S}$ lines are connected to the Vero board. The sampled parameter values are converted into actual parameter values and then by using the AT commands are sent from the Arduino to the ESP8266 WiFi module. The ESP8266 Wi-Fi module can make use of a home router to make internet connection to the cloud based ThingSpeak. The data is received when you log in to ThingSpeak and then displayed and analyzed.

There are two types of temperature sensors used in this design. The DHT11 which is a temperature and humidity sensor is used to measure the ambient room temperature and 
room humidity. The second sensor is DS18B20 which is a waterproof temperature sensor can be used for measuring temperature in a marine aquarium. Both sensors return an analogue value which is converted to actual parameter values by using the ADC conversion formula within the Arduino. The water level sensor is shown as a switch in Figure 4. The output of the water level sensor is low when the water level is below the sensor and high when the water level passes sensor. The relays are used to switch ON and OFF the following components, heater, cooling fan and the LED light. The three LED lights are used to show when these components are switched $\mathrm{ON}$ and the device is operational. The relays get their voltage source from the $V_{D D}$ and $V_{S S}$ connection on the board. The digital lines from the Arduino are used to control the relays that is switch them $\mathrm{ON}$ and OFF when necessary. This control is done in the software code of the Arduino. The connectivity of all these devices is summarized in Figure 4.

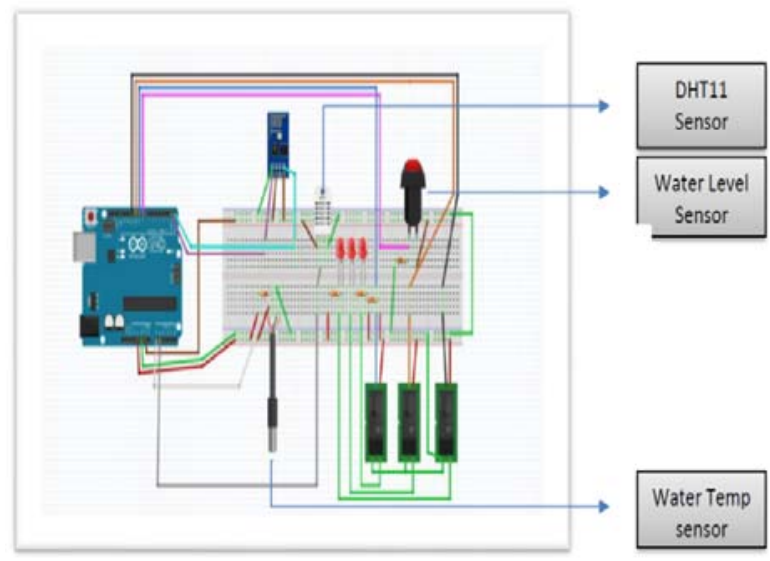

Fig. 4 Arduino with Temperature Sensors and ESP8266 Wi-Fi module

The wiring layout of the circuit diagram that can be used for PCB connection when building the final prototype can be seen in Figure 5.

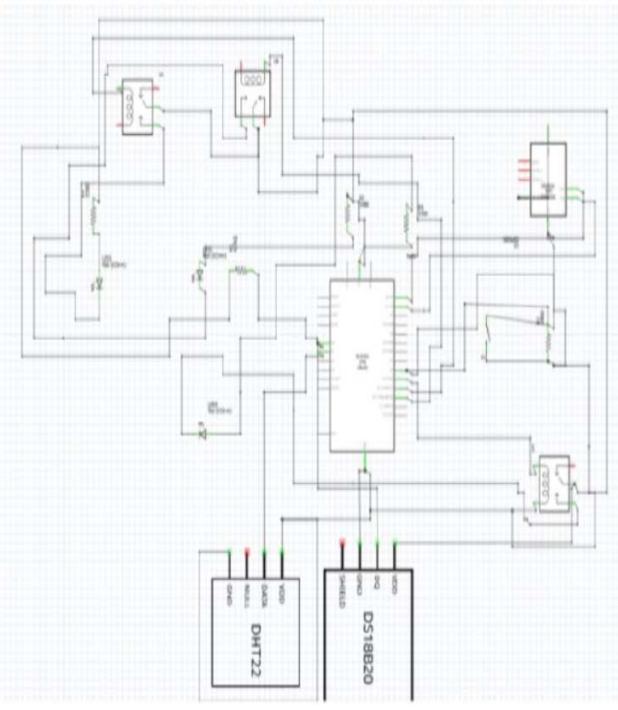

Fig. 5 Arduino with sensors and ESP8266 Wi-Fi module wiring layout
The Arduino headers are connected to a Veroboard and from the Veroboard to different components, this is done in the final prototype. The final boxed in prototype can be seen in Figure 6.

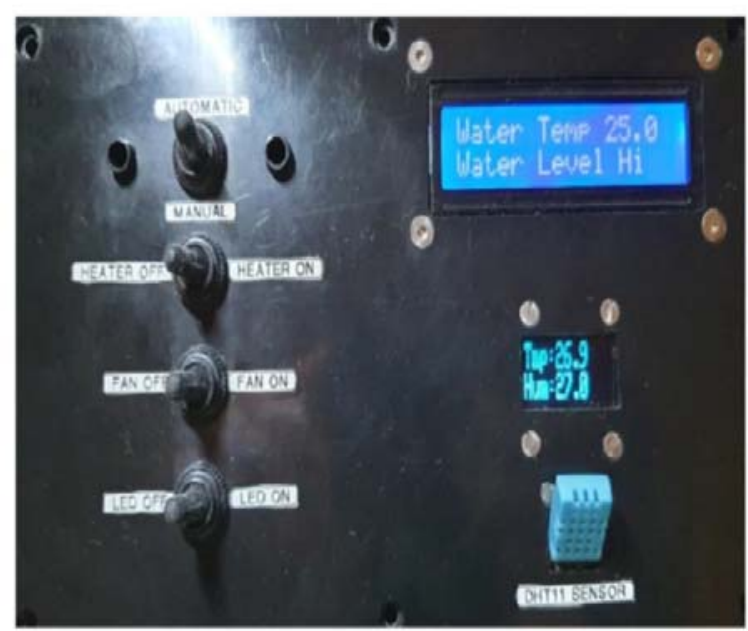

Fig. 6 Final design marine aquarium temperature controller

The internal connection that is headers, power supply, ESP8266 Wi-Fi module, Arduino Uno and relay module can be seen in Figure 7.

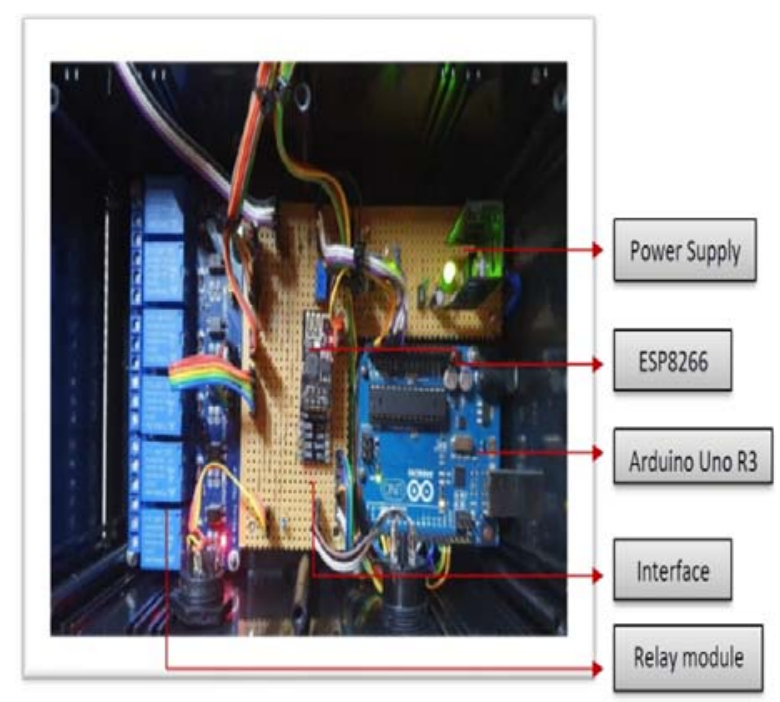

Fig. 7 Internal connection of the final prototype

The data read from the sensors consist of the ambient room temperature, humidity, aquarium water temperature and water level. These data is transmitted from the Arduino Uno to the cloud based ThingSpeak [10-14] via the ESP8266 Wi-Fi module and home router. The data displayed on the dashboard in ThingSpeak can be tracked or monitored over time as can be seen in Figure 8. 


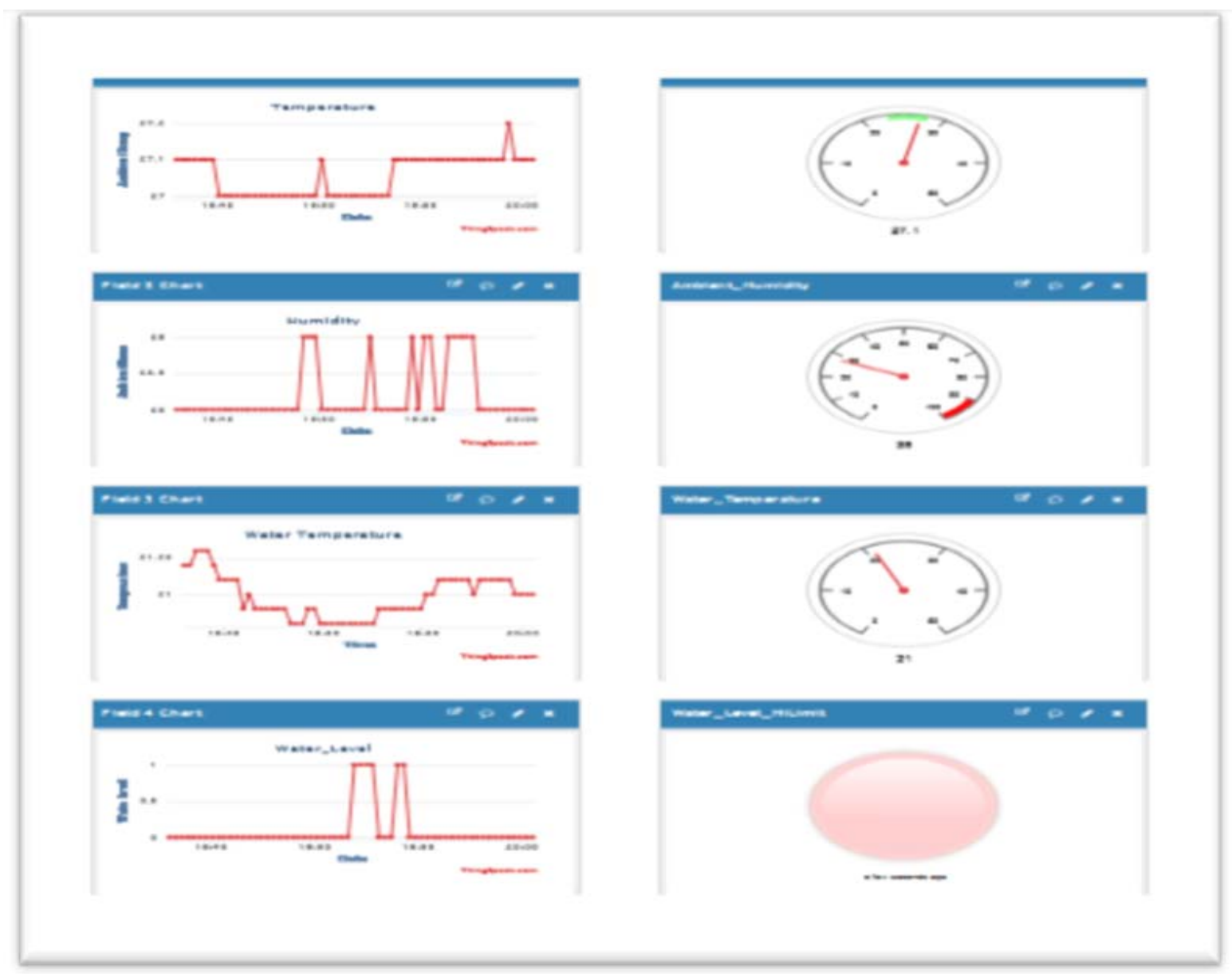

Fig. 8 ThingSpeak Realtime data on the IoT

Figure 8 shows the variations in ambient room temperature, humidity, aquarium water temperature and aquarium water level in real time. The aquarium water temperature initially drops gradually with respect to time and then with some heating rises again. The ambient temperature also follows a similar trajectory.

\section{CONCLUSION}

This paper focuses on the design of a marine aquarium temperature controller using the Ardunio Uno. The controller measures the aquarium water temperature, water level, ambient room temperature and humidity. The measured data is sent to the internet cloud server which hosts the ThingSpeak application via the ESP8266 Wi-Fi module and a home router. The data can be viewed and analyzed on ThingSpeak in real time. The data is recorded on ThingSpeak and can also be exported to a csv file for archiving. In addition, the data is also displayed on an LCD and OLED displays for immediate viewing by the user. The LCD shows the aquarium water temperature and the water levels while the OLED display shows the ambient room temperature and humidity. There are manual switches on the prototype that can be used to switch $\mathrm{ON}$ and OFF the heater, cooling fan and LED lights when it is required to control the variable of aquarium water temperature and indicate the device is operational. This device is useful for marine aquarium applications and connection to the IoT to receive data online. The prototype ideas can be applied to other fields of investigation where data needs to be collected from a remote source and made available for analysis and interpretation anywhere on the internet.

\section{ACKNOWLEDGMENT}

We would like to acknowledge the University of South Africa and the ESKOM South Africa TESP Grant for assistance with funding towards the research and provision of resources to complete the project.

\section{REFERENCES}

[1] Q. A. Mahmood, A. T. Nawaf, M. N. Esmael, L.T Abdulateef and O.S. Dahham, "PID Temperature Control of Demineralized Water Tank," IOP Conf. Series: Material Science and Engineering, vol. 454, pp. 012031, 2018, doi:10.1088/1757-899X/454/1/012031.

[2] J. Ding, M. Nemati, C. Ranaweer and J. Choi, "IoT Connectivity Technologies and Applications: A Survey", IEEE Access, Vol. 8, pp. 67646-67673, 06 April 2020, doi: 10.1109/ACCESS.2020.2985932.

[3] J. M. Yeom, H.J. Jung, S.Y. Choi, D.S. Lee and S.R. Lim, "Environmental Effects of the Technology Transition from LiquidCrystal Display (LCD) to Organic Light-Emitting Diode (OLED) Display from and E-Waste Management Perspective", International Journal of Environmental Research, vol. 12, pp. 479-488, 2018, doi: 10.1007/s41742-018-0106-y. 
[4] K. Jones, L. Liu, "What Where Wi: An Analysis of Millions of WiFi Access Points", IEEE International Conference on Portable Information Devices, 25-29 May, 2007, ISBN 1-4244-1039-8, doi: 10.1109/PORTABLE.2007.45.

[5] A. Priyati, M. Sahbandi, GMD. Putra and D.A Setiawati, "The Design of Automatic Sprinkler based on Arduino Uno Microcontroller", IOP Conference Series: Earth and Environmenta Science, vol. 355, pp. 012088, The $3^{\text {rd }}$ International Symposium on Agricultutral and Biosystem Engineering, 6- $8^{\text {th }}$ August 2019, doi: $10.1088 / 1755-1315 / 355 / 1 / 012088$

[6] Z. W. Hu, " $I^{2} C$ Protocol Design for Reusability" Third International Symposium on Information Processing, Qingdao, China, 15-17 October 2010, ISBN 978-1-4244-8627-4, doi: 10.1109/ISIP.2010.51

[7] U. Jovanovic, I. Jovanovic and D. Mancic, "Overview of Temperature Sensors for Temperature Measurement of PV Modules", Telecommunications Forum (TELFOR), Belgrade, Serbia, 20-21 Nov, 2018, ISBN 978-1-5386-7171-9, doi: 10.1109/TELFOR.2018.8612096.

[8] A. Abdelmoumene and H. Bentarzi, "A review on protective relays' development and trends", Journal of Energy in Southern Africa, vol. 25, no. 2, pp. 91-95, Cape town, May 2014, Available online: http://www.scielo.org.za/pdf/jesa/v25n2/10.pdf

[9] S. Ovcharov, S. Vichev and P. Yakimov, "Investigation on protective relays testing methods", $24^{\text {th }}$ International spring Seminar on Electronics Technology. Concurrrent Engineering in Electronic Packaging, ISSE, 5-9 th $^{\text {th }}$ May 2001, Romania, ISBN: 07803-7111-9, doi: 10.1109/ISSE.2001.931069

[10] M. P. Raju and A. J. Laxmi, "IOT based Online Load Forecasting using Machine Learning Algorithms", Third International Conference on Computing and Network Communications
(CoCoNet'19), Procedia Computer Science, vol. 171, pp. 551-560, 2020.

[11] N. Penchalaiah, J. N. Emmanuel, S.S. Kamal and C.V.L. Narayana "IoT Based Smart Farming Using Thingspeak and MATLAB", $3^{\text {rd }}$ International Conference on Communications and Cyber Physical Engineering ICCCE, pp. 1273-1295, $12^{\text {th }}$ October 2020, Available online: https://link.springer.com/chapter/10.1007\%2F978-981-157961-5 117.

[12] S. Faiazuddin, M.V. Lakshmaiah and K. T. Alam, "Design and Implementation of IoT based Indoor Air Quality Monitoring and control system", Solid State Technology, vol. 63, No. 2, 2020. Available

Online: http://solidstatetechnology.us/index.php/JSST/article/view/3956.

[13] H.A. Kusuma, R. Anjasmara, T. Suhendra, H. Yunianto and S. Nugraha, "An IoT Based Coastal Weather and Air Quality Monitoring Using GSM Technology", Journal of Physics: Conference Series, vol. 1501, pp. 012004, 2-3 ${ }^{\text {rd }}$ November 2019, Yogyakarta, Indonesia, doi: 10.1088/1742-6596/1501/1/012004.

[14] A. Wazwaz and K. Amin, "Error Rate Control in Humidity and Temperature Sensors Using IoT and ThingSpeak", $10^{\text {th }}$ International Conference on Information Communication and Management, ICICM, August 2020, pp. 71-75, doi: 10.1145/3418981.3418993.

[15] D. Parida, A. Behera, J.K. Naik, S. Pattanaik and R. S. Nanda, "Realtime Environment Monitoring System using ESP8266 and ThingSpeak on Interent of Things Platform", International Conference on Intelligent Computing and Control Systems (ICCS), $15-17^{\text {th }}$ May 2019, Madurai, India, ISBN 978-1-5386-8113-8, doi: $\underline{10.1109 / \text { ICCS45141.2019.9065451 }}$ 